\title{
Multi-feature Intensity Inhomogeneity Correction in MR Images
}

\author{
Uroš Vovk, Franjo Pernuš, and Boštjan Likar \\ Faculty of Electrical Engineering, Tržaška 25, 1000 Ljubljana, Slovenia \\ \{uros.vovk, franjo.pernus, bostjan.likar\}@fe.uni-lj.si
}

\begin{abstract}
In MRI, image intensity inhomogeneity is an adverse phenomenon that increases inter-tissue overlapping and hampers quantitative analysis. This study provides a powerful fully automated intensity inhomogeneity correction method that makes no a prior assumptions on the image intensity distribution and is able to correct intensity inhomogeneity with high dynamics. Besides using intensity features, as in most of the existing methods, spatial image features are also incorporated into the correction algorithm. A force is computed in each image point so that distribution of multiple features will shrink in the direction of intensity feature. Extensive regularization of those forces produces smooth inhomogeneity correction estimate, which is gradually improved in an iterative correction framework. The method was tested on simulated and real MR images for which gold standard segmentations were available. The results showed that the method was successful on uniform as well as on low and highly dynamic intensity uniformity images.
\end{abstract}

\section{Introduction}

Image intensity inhomogeneity, also referred to as bias field, intensity nonuniformity or shading, is perceived as a smooth intensity variation across the image. In MRI, intensity inhomogeneity may be caused by a number of factors, such as poor radio frequency coil uniformity, static field inhomogeneity, radio frequency penetration, gradient-driven eddy currents, and overall patient anatomy and position $[1,2]$. The problem is especially cumbersome because inhomogeneity depends on the measured object and therefore cannot be eliminated or reduced by scanner calibration. A common solution is to conduct retrospective inhomogeneity correction, which is a necessary pre-processing step in many automatic image analysis tasks, e.g. in segmentation or registration, and especially if quantitative analysis is the final goal.

The most intuitive approach for intensity inhomogeneity correction is image smoothing or homomorphic filtering [3, 4, 5]. These methods are based on the assumption that intensity inhomogeneity is a low frequency signal which can be suppressed by high pass filters. However, since the imaged objects themselves usually contain low frequencies, filtering methods often fail to produce meaningful correction. Iterative optimisation that maximises the frequency content of the distribution of tissue intensity has been proposed in [6]. Dawant et al. [7] manually selected some points inside white matter and estimated the bias field by fitting splines to the intensities of these points. Styner et al. [8] modelled each tissue simply by its 
mean intensity value. A parametric bias field is fitted to the image in an iterative process, which requires initialisation in a form of class means and special masks that have to be defined for each type of images. Information minimisation technique [9] has proved to be very robust and accurate approach in which bias field is modelled by polynomials and applied to acquired image in an optimisation process. The method based on fuzzy C-means [10] minimises the sum of class membership function and the first and second order regularisation terms that ensure the smooth bias field. Ahmed et al. [11] used a fuzzy C-means algorithm and combined bias field with adaptive segmentation. Other segmentation-based methods use expectationmaximisation algorithm to compute the bias field from the residue image by either spatial filtering [12] or by weighted least-squares fit of the polynomial bias model [13]. These methods interleave classification and bias field estimation [12] and also the estimation of class-conditional intensity distribution [13] but require initialisation, which is not trivial and practical. Besides, the assumption on normality of intensity distribution of individual tissues may often not be valid, especially when correcting pathological data.

In this paper we propose a novel fully automated bias correction method that makes no assumption on the distribution of image intensities and provide nonparametric correction. As most of the existing methods, the method is based on intensity features but also additional spatial image features are incorporated to improve bias correction and to make it more dynamic.

\section{Method}

Corruption of intensity homogeneity in MR imaging is a multiplicative phenomenon that is most commonly described by the following model,

$$
u(x)=v(x) s(x)+n(x),
$$

in which the acquired image $u(x)$ is a combination of the uncorrupted image $v(x)$, corrupted by the multiplicative bias field $s(x)$, and statistically independent noise $n(x)$. The problem of correction of intensity inhomogeneity is to estimate $v(x)$, given the acquired image $u(x)$.

The proposed method can be outlined in four steps:

S1. Calculate probability distribution $p($.) of image features

S2. Estimate the forces that will shrink $p($.) in the direction of intensity feature

S3. Estimate the bias correction field

S4. Perform partial correction and stop if predefined number of iterations is reached. Otherwise go to S1.

The above steps are described in more detail in the following sections. 


\subsection{Probability Distribution Calculation}

Probability distribution of image features $p(i, d)$ is determined by binning the intensities $(i)$ and the corresponding second spatial derivatives $(d)$ into a discrete twodimensional feature space. Second derivatives are obtained by convoluting the original image by the Laplacian operator, which was implemented as a $3 \times 3$ kernel and applied separately for each slice.

The purpose of using additional image features in a form of second derivatives is to additionally separate tissue clusters in the feature space [14]. This is especially important when image intensities are not enough discriminating features, i.e. when intensity distributions of distinct tissues overlap significantly. This adverse and unfortunately quite frequent phenomenon in MRI is the main source of error in segmentation and inhomogeneity correction methods.

\subsection{Force Estimation}

In order to obtain an estimation of forces $F_{x}$ for each image point $x$ that will shrink the probability distribution $p(i, d)$ of an image $u(x)$ in the direction of intensity feature $i$, we first define a global energy, say $E$, as a measure of probability distribution dispersion. For this purpose we use the Shannon entropy $H$,

$$
E=H(u(x))=-\sum_{i, d} p(i, d) \log p(i, d) .
$$

The entropy $H$ is usually computed as above by summation of the uncertainties log $p(i, d)$ in the feature space domain but since we are seeking a contribution of each image point $x$ to the global entropy we will compute the entropy alternatively by summation of the uncertainties over the image domain $x$ of a size $X$,

$$
E=H(u(x))=-\frac{1}{X} \sum_{x} \log p(i(x), d(x)) .
$$

We can now derive point energy, say $E_{x}$, which is the contribution of each image point $x$ to the global energy $E$,

$$
E_{x}=-\frac{1}{X} \log p(i(x), d(x)) .
$$

$E_{x}$ is, therefore, a contribution of image point $x$ to the dispersion of probability distribution $p(i, d)$. Corresponding point force $F_{x}$ that will shrink the probability distribution $p(i, d)$ in the direction of intensity feature $i$ can, thus, be obtained by deriving the point energy $E_{x}$ over the intensities and changing the sign to plus,

$$
F_{x}=\frac{1}{X} \frac{\partial}{\partial i}(\log p(i(x), d(x))) .
$$

Forces $F_{x}$ are computed by a Sobel operator in the feature space for all feature pairs $(i, d)$ and then mapped to the points with corresponding features in the image space. The obtained forces can be viewed upon as votes for point intensity changes that would result in less disperse probability distribution. 


\subsection{Bias Correction Estimation}

To control the speed of the iterative bias correction and to make it independent of the shape of feature probability distributions, point forces $F_{x}$ are first normalized in magnitude. The normalized forces are then regularized in the image domain by a sufficiently wide Gaussian kernel $g$. This yields smooth field of normalized forces that is used to derive an estimation of multiplicative bias correction field $s^{-1}(x)$,

$$
s^{-1}(x)=1+\left(f \frac{F_{x}}{\mu\left(\left|F_{x}\right|\right)}\right) * g,
$$

where $\mu\left(\left|F_{x}\right|\right)$ denotes mean of absolute force, $f$ a predefined magnitude of forces, and $*$ a convolution. A degree of smoothness of bias correction field $s^{-1}(x)$ is determined by the standard deviation $\sigma_{g}$ of the Gaussian kernel $g$.

\subsection{Partial Correction}

Partial bias correction is performed in each iteration, say $n^{\text {th }}$, by applying bias correction field $s^{-1}(x)$ to the input image $u_{n}(x)$ so that mean intensity (brightness) and standard deviation (contrast) of the input image are preserved,

$$
u_{n+1}(x) \Leftarrow u_{\sigma}(x)+\mu\left(u_{0}(x)\right)-\mu\left(u_{\sigma}(x)\right), u_{\sigma}(x)=u_{n}(x) s^{-1}(x) \frac{\sigma\left(u_{0}(x)\right)}{\sigma\left(u_{n}(x) s^{-1}(x)\right)} .
$$

The size of partial correction depends on the predefined force magnitude $f$ and the standard deviation $\sigma_{g}$ that controls the regularization step, i.e. the smoothness of the bias correction field.

\section{Results}

Removal of background in MRI images is essential in nearly all methods because background represents air voxels that are not corrupted by the multiplicative bias field and would therefore hamper inhomogeneity correction of the neighbouring tissue voxels. To mask out dark air voxels we remove all voxels with intensities smaller than the predefined intensity threshold, which is the simplest and most commonly used approach $[9,6,13]$.

To demonstrate and evaluate the performance of proposed method, we applied it to several simulated and real magnetic resonance images of the human brain.

In the first set of images, variations of digital brain phantom acquired from Brainweb-MRI Simulator [15] were considered: six pairs of volumes with 3\% Gaussian noise, including T1-, T2- and PD-modalities, normal and Multiple Sclerosis (MS) lesion cases. Each pair comprised shading free volume and its corrupted version with $40 \%$ intensity inhomogeneity. The resolutions were $181 \times 217 \times 181$ voxels, sized $1 \times 1 \times 1 \mathrm{~mm}^{3}$.

In the second set of images, extra bias fields with higher degree of dynamics were added to the images from the first set. Bias fields were generated by cubic B-spline 
interpolation between equally spaced nodes at every 50 voxels in each direction. Node values (multiplication factors) were randomly distributed on the interval between 0.8 and 1.2.

Third set consisted of six volumes, three of a normal volunteer $(256 \times 256 \times 25$ voxels 8 bit $)$ and three of a tumour patient $(256 \times 256 \times 20$ voxels, 8bit, slice thickness of $5 \mathrm{~mm}$, in plane resolution of $1 \times 1 \mathrm{~mm})$. White and grey matter manual segmentations were available [16].

Volumes of the second set were used to show the capability of our method to successfully cope with bias fields, which have high degree of dynamics. Changing the size of the regularization kernel has a direct impact on the estimated fields. Reducing kernel size makes the fields more locally flexible, but looses a part of its regularization functionality. The effect of regularization kernel size on the shape of reconstructed bias field is shown in Fig. 1. Image (a) indicates the position of the bias field profiles shown on the images (b) and (c). Solid lines indicate generated bias field and broken lines the corresponding reconstruction after inhomogeneity correction. Standard deviations of the regularization kernels were set to $30 \mathrm{~mm}$ in case (b) and to $90 \mathrm{~mm}$ in case (c). In the first case high correlation between applied and reconstructed bias fields was found, while the in second case the correlation was poor due to too high regularization.

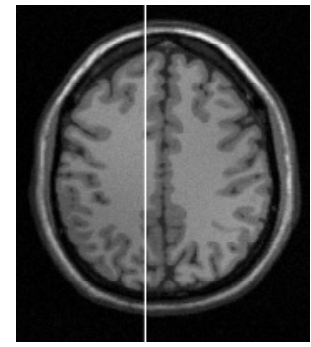

a

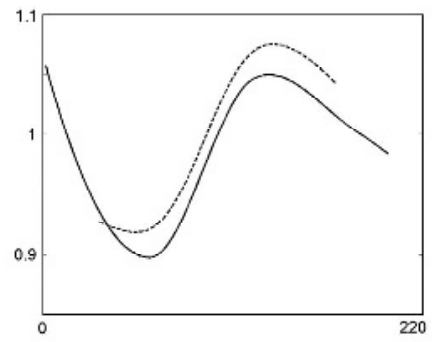

$\mathrm{b}$

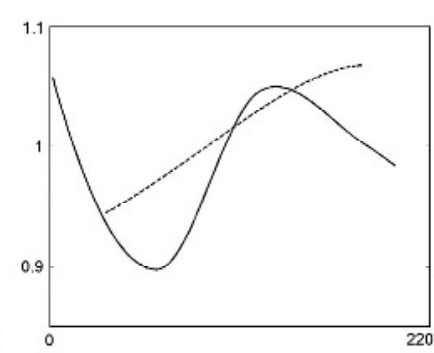

c

Fig. 1. Image (a) shows the profile position, (b) and (c) the applied (solid curve) and reconstructed bias field (broken curve) for the regularization of 30 and $90 \mathrm{~mm}$, respectively

Quantitative evaluation was performed by computing the coefficient of joint variations (cjv) [9] between grey (GM) and white matter (WM) of the brain, which were segmented in all three sets of images. $C j v$ is computed from standard deviations $(\sigma)$ and mean values $(\mu)$ of the voxel intensities belonging to the two matters,

$$
\operatorname{cjv}(G M, W M)=\frac{\sigma(G M)+\sigma(W M)}{|\mu(G M)-\mu(W M)|} .
$$

The measure is independent of the changes in contrast and brightness and measures the intensity inhomogeneity in the sense of minimizing the intensity overlapping between two tissues.

In Table 1 the results of inhomogeneity correction in the first set of images are given. The four columns describe the volume properties (modality, bias field and pathology) and starting, final and ideal $c j v$ values. The method successfully corrected 
the first six volumes and did not induce any artefacts into the second half of the inhomogeneity free volumes.

Table 1. Coefficient of joint variations of grey and white matter for the first dataset

\begin{tabular}{llll}
\hline Volume & $c j v_{\text {start }}[\%]$ & $c j v_{\text {end }}[\%]$ & $c j v_{\text {ideal }}[\%]$ \\
\hline T1 40\% & 69.3 & 51.7 & 51.6 \\
T2 40\% & 106.4 & 83.4 & 83.2 \\
PD 40\% & 163.0 & 62.8 & 64.9 \\
T1 40\% MS lesions & 68.0 & 51.0 & 50.9 \\
T2 40\% MS lesions & 123.8 & 74.5 & 74.9 \\
PD 40\% MS lesions & 195.6 & 64.9 & 66.9 \\
T1 0\% & 51.6 & 51.9 & 51.6 \\
T2 0\% & 83.2 & 83.4 & 83.2 \\
PD 0\% & 64.9 & 65.5 & 64.9 \\
T1 0\% MS lesions & 50.9 & 51.2 & 50.9 \\
T2 0\% MS lesions & 74.9 & 75.0 & 74.9 \\
PD 0\% MS lesions & 66.9 & 67.7 & 66.9 \\
\hline
\end{tabular}

Table 2. Coefficient of joint variations of grey and white matter for the second dataset

\begin{tabular}{llll}
\hline Volume $(\mathrm{df}-$ dynamic field $)$ & $c j v_{\text {start }}[\%]$ & $c j v_{\text {end }}[\%]$ & $c j v_{\text {ideal }}[\%]$ \\
\hline T1 $40 \% \times \mathrm{df}$ & 86.8 & 51.7 & 51.6 \\
T2 $40 \% \times \mathrm{df}$ & 107.7 & 84.5 & 83.2 \\
PD $40 \% \times \mathrm{df}$ & 228.5 & 64.7 & 64.9 \\
T1 40\% MS lesions $\times \mathrm{df}$ & 84.8 & 51.0 & 50.9 \\
T2 40\% MS lesions $\times \mathrm{df}$ & 128.1 & 75.8 & 74.9 \\
PD 40\% MS lesions $\times \mathrm{df}$ & 276.3 & 66.6 & 66.9 \\
T1 0\% $\times$ df & 78.5 & 51.9 & 51.6 \\
T2 0\% $\times$ df & 93.1 & 84.7 & 83.2 \\
PD 0\% $\times$ df & 125.6 & 67.2 & 64.9 \\
T1 0\% MS lesions $\times$ df & 76.6 & 51.2 & 50.9 \\
T2 0\% MS lesions $\times$ df & 98.7 & 76.6 & 74.9 \\
PD 0\% MS lesions $\times$ df & 141.8 & 69.4 & 66.9 \\
\hline
\end{tabular}

The results of inhomogeneity correction in the second set of images are given in Table 2. The proposed method successfully corrected the inhomogeneities with high dynamics. Final $c j v$ values did not match the ideal ones as perfectly as in the first set, because the induced bias fields could not be exactly reconstructed by Gaussian convolution. Parameters were fixed for all experiments with $f=0.02, \sigma_{g}=30 \mathrm{~mm}$, number of iterations was 30. Size of the histogram was $256(i)$ by $400(d)$ bins.

Table 3 shows the results on real MR volumes of the third set. The volumes without intensity inhomogeneity were not known so that the extent of the achieved correction cannot be determined. The Last column states relative change of $c j v$ value, where it can be seen that improvement was achieved in all volumes. 
Table 3. Coefficient of joint variations of grey and white matter for the real volumes

\begin{tabular}{llll}
\hline Volume & $c j v_{\text {sart }}[\%]$ & $c j v_{\text {end }}[\%]$ & $c j v_{\text {change }}[\%]$ \\
\hline T1 normal & 138.0 & 132.6 & -3.9 \\
T2 normal & 90.7 & 89.0 & -1.9 \\
PD normal & 77.8 & 70.4 & -9.6 \\
T1 tumour & 175.7 & 169.4 & -3.6 \\
T2 tumour & 169.3 & 159.6 & -5.7 \\
PD tumour & 133.8 & 117.3 & -12.4 \\
\hline
\end{tabular}

\section{Conclusion}

The proposed fully automated bias correction method makes no assumption on the distribution of image intensities and provides non-parametric correction. No a priori knowledge such as digital brain atlases or reference points is needed. Spatial image features are incorporated in addition to commonly used intensity features, which give the method enough information to successfully correct even highly dynamic bias fields.

The method performed well on all tested images. The second set of images with artificially generated dynamic bias fields showed a possible advantage over many other methods that incorporate more rigid correction models. The performance of the proposed method depends on the overlap between the probability distributions of image features, which should be small. The proposed correction framework, however, enables straightforward incorporation of additional image features that could yield additional discriminative power and improve the correction performance on the images of poor quality.

Acknowledgement. This work has been supported by the Slovenian Ministry of Education, Science and Sport under grant P2-0232.

\section{References}

1. Condon, B. R., Patterson, J., Wyper, D., Jenkins, A.,Hadley, D. M.: Image non-uniformity in magnetic resonance imaging: its magnitude and methods for its correction. The British Journal of Radiology 60 (1987) 83-87

2. Simmons, A., Tofts, P. S., Barker, G. J.,Arridge, S. R.: Sources of intensity nonuniformity in spin echo images at 1.5 T. Magn Reson Med 32 (1994) 121-128

3. Johnson, B., Atkins, M. S., Mackiewich, B.,Anderson, M.: Segmentation of multiple sclerosis lesions in intensity corrected multispectral MRI. IEEE transaction on medical imaging 15 (1996) 154-169

4. Koivula, A., Alakuijala, J.,Tervonen, O.: Image feature based automatic correction of lowfrequency spatial intensity variations in MR images. Magn Reson Imaging 15 (1997) $1167-1175$

5. Haselgrove, J.,Prammer, M.: An algorithm for compensating of surface-coil images for sensitivity of the surface coil. Magnetic Resonance Imaging 4 (1986) 469-472 
6. Sled, J. G., Zijdenbos, A. P.,Evans, A. C.: A nonparametric method for automatic correction of intensity nonuniformity in MRI data. IEEE Trans Med Imaging 17 (1998) 87-97

7. Dawant, B. M., Zijdenbos, A. P.,Margolin, R. A.: Correction o fintensity variations in MR images for computer-aided tissues classification. IEEE Trans Med Imaging 12 (1993) 770781

8. Styner, M., Brechbuhler, C., Szekely, G.,Gerig, G.: Parametric estimate of intensity inhomogeneities applied to MRI. IEEE Trans Med Imaging 19 (2000) 153-165

9. Likar, B., Viergever, M. A.,Pernuš, F.: Retrospective correction of MR intensity inhomogeneity by information minimization. IEEE Trans Med Imaging 20 (2001) 13981410

10. Pham, D. L.,Prince, J. L.: Adaptive fuzzy segmentation of magnetic resonance images. IEEE Trans Med Imaging 18 (1999) 737-752

11. Ahmed, M. N., Yamany, S. M., Mohamed, N., Farag, A. A.,Moriarty, T.: A modified fuzzy C-means algorithm for bias field estimation and segmentation of MRI data. IEEE Trans Med Imaging 21 (2002) 193-199

12. Wells III, W. M., Grimson, W. E. L.,Jolezs, F. A.: Adaptive segmentation of MRI data. IEEE Trans Med Imaging 15 (1996) 429-442

13. Van Leemput, K., Maes, F., Vandermeulen, D.,Suetens, P.: Automated model-based bias field correction of MR images of the brain. IEEE Trans Med Imaging 18 (1999) 885-896

14. Derganc, J., Likar, B., Pernuš, F.: Nonparametric segmentation of multispectral MR images incorporating spatial and intensity information. In: Sonka, M., Fitzpatrick, J.M. (eds.): Proceedings of SPIE Medical Imaging 2002: Image processing, Vol. 4684. SPIE Press, San Diego USA (2002) 391-400

15. Cocosco, C. A., Kollokian, V., S, K. R. K.,Evans, A. C.: BrainWeb: Online Interface to a 3D MRI Simulated Brain Database. NeuroImage 5 (1997) 425-425

16. Velthuizen, R. P., Clarke, L. P., Phuphanich, S., Hall, L. O., Bensaid, A. M., Arrington, J. A., Greenberg, H. M.,Silbiger, M. L.: Unsupervised measurement of brain tumor volume on MR images. J Magn Reson Imaging 5 (1995) 594-605 